present, definite reaction; phosphates, trace present; sulphates, doubtful, perhaps a very slight trace; urea present; sugar not present in measurable amount; cholesterol not present; blood pigment, examination by spectroscope failed to reveal absorption bands. On the whole, the composition of the fluid bears a definite resemblance to that of lymph." The fluid was sterile.

Microscopical examination of the cyst wall.-The interior of the cyst is lined by a stratified epithelium, which is clearly seen in both micro-photographs. The sections were stained by logwood and eosin or van Gieson's stain. Oval nuclei are seen arranged in several layers at some parts and in one or two layers at other parts. There is little or no indication of the cell wall. External to the epithelium is dense fibrous tissue forming a thick layer and containing some blood-vessels. This is separated by a looser tissue from the conjunctival epithelium. In sections stained by Weigert's elastic tissue stain numerous wavy dichotomous branching fibres are seen, mainly in the tissue between the dense fibrous tissue and the conjunctival epithelium. There is no trace of any foreign body, hairs or glands. As regards the aetiology of scleral cysts reference may be made to Goulden and Whiting's paper and to the discussion that followed it. In the present case the cyst appears to have been present at the time of birth.

The photographs of the present case were kindly taken by Dr. William Brown, and the micro-photographs of the sections by Dr. Alexander Low.

\title{
FREQUENCY OF METASTATIC CARCINOMA OF THE CHOROID
}

BY

\section{H. USHER \\ ABERDEEN}

IN Volume VII, 1923, of this journal an account is given of three cases of metastatic carcinoma of the choroid, one in a woman with mammary cancer and the other two in males. Reference is made there to the apparent infrequency of such cases, and of 107 cases collected from the literature and tabulated at that time the majority of cases, numbering 60 , were single cases reported by 60 different observers. Fifteen other observers each recorded two cases, three other observers each reported three cases, and two others, Ginsberg and Fehr, have each recorded four cases. There is no record of five cases, or more, having been published by any one author. The conclusion, therefore, as judged by the number of reported 
cases, is that metastatic carcinoma of the choroid is evidently of infrequent occurrence. Since the above-mentioned paper was written another case has presented itself, short details of which are as follows : Mrs. M., aged 47 years, had an operation for cancer of her right breast in May, 1919. Four years later, May 16, 1923, her health was satisfactory, she had a good appetite and slept well, but she was evidently nervous and had some headache and giddiness in the morning. There had been difficulty in seeing for a few months and on examination a large retinal detachment was seen in the lower part of her right fundus oculi. The left fundus was normal. On July 26, she reported attacks of pain in the right eye which kept her awake at night. Vision in this eye was now reduced to perception of light, tension was +1 , and the anterior chamber was shallow. The left fundus was normal. Mr. Marnoch, professor of surgery, who had operated for her breast cancer, saw her again in November, 1923, when he found "recurrence on forehead, a bony nodule at lower end of right ulna -also one over lower part of back and almost certainly a spinal metastasis (pain extending down her legs)." She became very blind shortly before her death and the pupils were dilated though she was having large quantities of morphia, so it is probable that each choroid contained a tumour. In one-third of the cases of metastatic carcinoma of the choroid, a tumour occurs in both choroids. Death occurred on January 21, 1924. There was no post-mortem examination, but permission was obtained to remove the right eye which was excised and a flat carcinomatous growth was found in the choroid with its greatest thickness, $1.25 \mathrm{~mm}$., at the posterior part of the choroid. Towards the edge of the growth where the carcinomatous cells are arranged in columns only the outer part of the choroid is occupied by the tumour. The retina is completely detached except at the optic disc and ora serrata. The root of the iris is adherent to the cornea.

The detachment of the retina had first been observed eight months before death. The first evidence of metastasis was failing vision and this did not occur until some four years after the operation for the breast cancer.

There can be little doubt that, although there was no autopsy, a case seen in 1904 was one of metastatic carcinoma of choroid. A female, aged 42 years, complained of defective vision of her left eye; her left breast had been amputated for carcinoma two years previously; there was a diffuse grey swelling immediately below the left optic disc and macula which later extended to upper part of macula and was focussed with $+6 \mathrm{D}$., retinal vessels on the swelling were not tortuous. The liver became enlarged and was believed to contain carcinomatous growths. Death occurred six months after vision of the left eye was found to be defective. 